\title{
A Case Study Protocol for Outsourcing Contract Management Model (OCMM)
}

\author{
Abdul Wahid Khan ${ }^{2,}$, Siffat Ullah Khan ${ }^{1}$ and Fahim Khan ${ }^{1}$ \\ ${ }^{1}$ Department of Computer Science \& IT, University of Malakand, KPK, Pakistan. \\ ${ }^{2}$ Department of Computer Science \& IT, University of \& Technology, Bannu, KPK, Pakistan. \\ * Corresponding author. email: wahidkn@gmail.com \\ Manuscript submitted November 24, 2016; accepted February 15, 2017. \\ doi: $10.17706 /$ jsw.12.5.348-354
}

\begin{abstract}
Offshore software development is a software paradigm for developing high quality software at low wage country at reduces cost. We have proposed a model of outsourcing contract management model (OCMM). The success factors, risk factors and practices for the identified factors were identified through systematic literature review (SLR). Questionnaire survey was conducted for the validation of the SLR findings. This protocol developed for the evaluation of OCMM through case studies. This evaluation will assist in improvement of the propose model and will set according to requirement.
\end{abstract}

Keywords: Software outsourcing contract, vendor and case study protocol.

\section{Introduction}

A conduction of a systematic literature review of outsourcing literature, along with questionnaire survey in the field of outsourcing industry, shows the importance to design a model in order to help outsourcing practitioners in measuring the OSDO vendor status for outsourcing contract management at its various stages such as pre-contract, during-contract and post-contract. The main goal of this research project is to empirically find out point of views and experiences of outsourcing experts about OSDO and to formulate an outsourcing contract management model (OCMM) in order to help outsourcing vendor organisations in evaluating their status at various stages for outsourcing contract management. The OCMM will stand on an empirical analysis of outsourcing skillful people's experiences and sensing of factors that have a positive or negative effect on OSDO inaugurals. The model of OCMM will help outsourcing vendor organisations in assessing their strong and weak areas in terms of planning, implementing, and evaluating suitable strategies to affirm their OSDO actions.

This research work focus to reduce the distance between OSDO research and practice in such a way that is approachable to both OSDO experts and researchers. Most of the research work finished with producing models or frameworks, which never make it into industrial practice. It is anticipated that this work will mitigate this course in OSDO by describing a well-realized and rational outsourcing contract management model. The proposed model will assist the vendor organisation to implement it due to its valuable features and will avoid implementation of the new devalue model or framework, which is discarded once their utility is questioned.

\section{Research Question}


I have explained the objective of the case studies in this protocol in order to assist out emerging developed model of OCMM. The below defines research question excited the work mentioned in this report:

RQ. Is the OCMM robust and practically applicable in respect of evaluating organisations (vendor) in proper management of outsourcing contract for OSDO activities?

\section{OCMM Development Stages}

We have used six stages in OCMM development as shown in Figure 1. In the first stage we have defined the criteria for OCMM development. The motivation for setting these criteria derived from literature [1]-[4] and by a thoughtfulness of the Technology Acceptance Model [5], [6]. The following standards will be used.

- Ease of use: The aim of this criterion is to define such a model (OCMM) that can easily interpret by outsourcing practitioners, easy to use and easy understandable.

- User satisfaction: The aim of this criterion is to analyze the level of user satisfaction with the results of the OCMM

- Structure of the OCMM: This criterion examines the effectiveness of the different components of OCMM and to assert the dispersion of CSFs and CBs across it defined levels.

In the second stage the research question will be defined. In stage 3 the data will be collected through the SLR and in stage 4 the SLR finding will be evaluated through questionnaire survey. Stage 5 is the stage where rationalization and structuring of outcomes will be performed. The OCMM will be developed here on the basis of empirical findings. In the final stage the case studies will be conducted for the evaluation of OCMM model.

We have collected two types of data in this research work: Firstly, factors that can have convinced (critical success factors - CSFs) or negative (critical barriers - CBs) effect on OSDO clients in the selection of vendors for outsourcing contract agreement. Secondly, how the identified CSFs and CBs are implemented. An approach of a systematic literature review (SLR) process was used for the identification of factors [7, 8]. In order to know how to implement the CSFs and how to avoid the CBs, questionnaire survey will be conducted in the outsourcing industry to get the opinions of the software outsourcing experts. A case study approach will be used for evaluation of the OCMM.

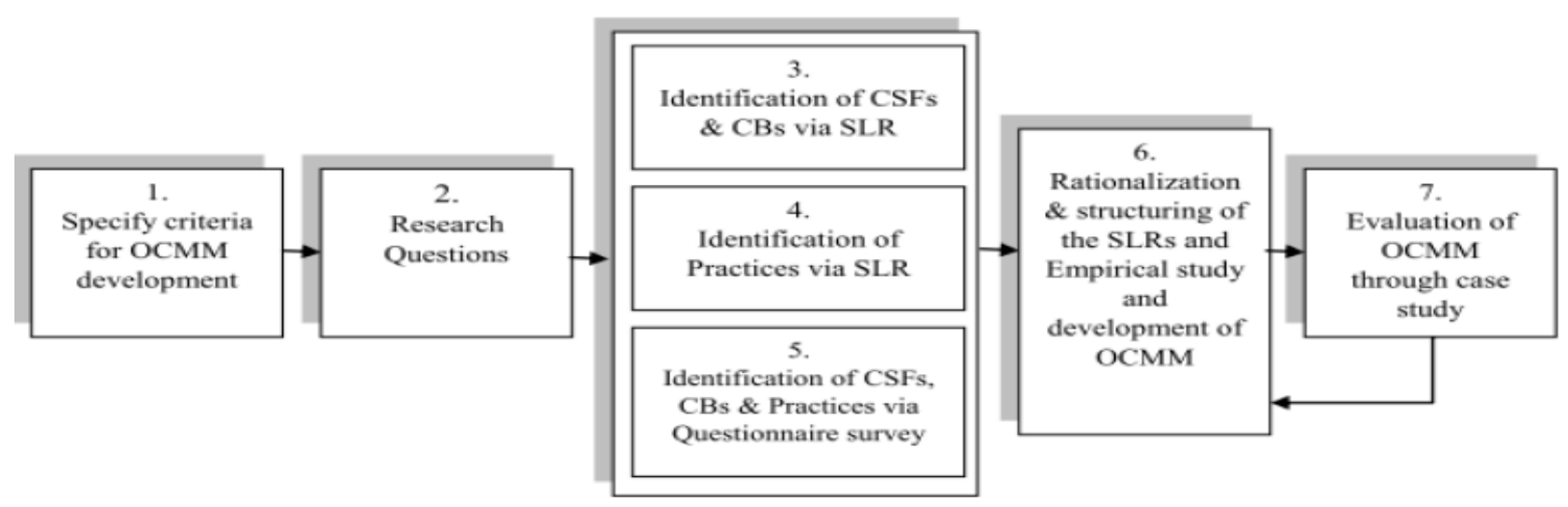

Fig. 1. OCMM development cycle activities.

\section{Research Plan}

\subsection{Data Collection and Analysis}

We have collected two types of data in this research work: Firstly, factors that can have convinced (critical success factors - CSFs) or negative (critical barriers - CBs) effect on OSDO clients in the selection of vendors 
for outsourcing contract agreement. Secondly, how the identified CSFs and CBs are implemented. We have used the same approach of CSFs as used by Rockart [9]. The CSFs and CBs were identified [10], [11] using the SLR process [7]. SLR is a systematic way used for exploring the primary studies for some specific research questions. SLR is different from ordinary literature review as it is formally planned and methodically accomplished. This method is less bias, but can increase the scientific values. SLR is a protocol base literature review, where first a protocol is developed, which provides a road map for future research.

A questionnaire survey will be conducted for the validation of identifying CSFs and CBs that a have positive or negative impact on client organisations in selection of outsourcing vendor organisation (s) that can execute an outsourcing contract decently. We will not only validate the SLR findings through questionnaire survey, but will also take opinions of software outsourcing experts about the solutions or guidelines or practices involved to implement these factors in OSDO vendor organisations.

\subsection{OCMM Assessment via Case Study}

For assessment of OCMM an approach of case study will be used. We will search for least four OSDO vendors' organisations for the assessment of the OCMM. The same approach is also used as reported in the literature for the assessment of SOVRM model [1] and [12]. The case study method of empirical study is considered a powerful evaluation tool and give useful real-world information [13], so we have planned to use this for OCMM assessment. According to Cooper and Schindler [14] the case study raises valuable insights for problem solving, rating and strategy. A feedback session will be conducted after running of case study with the participants, such that to get feedback about the pertinence of OCMM.

\subsection{Why to Use Case Study Approach}

As the OCMM development and application is in a real environment of software industry, so a case study research is considered to be an appropriate way for such kind of situation. Real life case studies are required because they:

- Will represent that OCMM is worthy and well fit in the real world environment.

- It will highlight the area of OCMM where requires some improvement.

- It will show the practicality and usability of the OCMM in use.

The assessment of product turns up the deficits area of the end product. The assessment helps in future planning and decision making. The finding of evaluation process assists to set the future directions.

\subsection{Companies' Identification for the Case Studies}

We have planned to find out the list of software outsourcing organisations from Pakistan Software Export Board. We will share a letter of invitation to these organisations to take part in the assessment of OCMM. We will conduct at least four case studies in OSDO vendor organizations.

\subsection{Criteria for the Case Studies}

We have planned to conduct at least four case studies at OSDO vendor organisations for the better improvement of the OCMM. The organisation selection will take part on the basis of their reputation in software outsourcing development projects and to release the case studies outcomes.

The participant of the case studies will be selected by the management of each vendor organisations. A communication between the parties will be made through email and face-to-face meeting for the duration of at least one month, such that to get a complete understanding of the OCMM.

We will communicate the relevant documents of OCMM before conduction of the case studies with the participant through email and will share the summary of the projects along with concerned documents. The role of each participant in the case studies will be described. A short training of some hours will be given to 
participants about the OCMM process understanding. In these trainings, different components of the OCMM will be explained and participants will be encouraged to use the OCMM independently without any hints or help from the researcher.

During each case study, the selected participants will encourage to use OCMM and will evaluate the level of software outsourcing management of their organisation independently without and suggestion or assistance from other researchers. The participant of same company consults with other colleagues during the evaluation such that to find the representative results of OCMM. Furthermore, participant of each case study will do evaluate based on observations and experience of previous projects.

We have motivated, due to the following criteria for the evaluation of the OCMM. The motivation for setting these criteria derived from literature [1]-[4] and by a thoughtfulness of the Technology Acceptance Model $[5,6]$. The following standards will be used.

- Ease of use: The aim of criterion is to define such a model (OCMM) that can easily interpret by outsourcing practitioners, easy to use and easy understandable.

- User satisfaction: The aim of this criterion is to analyze the level of user satisfaction with the results of the OCMM.

- Structure of the OCMM: This criterion examines the effectiveness of the different components of OCMM and to assert the dispersion of CSFs and CBs across it defined levels.

The above defined assessment criteria are enough for OCMM evaluation, because these criteria can be used to assess the effectiveness and quality of the product and can help to show up areas with any deficit. A feedback session will be conducted at the end case study with participant to get feedback the reliability of the OCMM. The feedback session will collect data in three areas: ease of use, user satisfaction and structure of OCMM. The questionnaire, developed for feedback session will be used as a means to structure the feedback session. There are four parts of questionnaire, i.e. demographic part, ease of use, user satisfaction and structure of the OCMM. Each feedback session will be an informal discussion and the questionnaire will be filled out by each participant on the spot. Each questionnaire will be qualitatively analysed.

\section{Analysis}

We have planned to analyse the collected data through case studies in the following ways:

- To evaluate the status of each vendor organisation for execution of outsourcing contract

- To assess a score for each CSF and CB at each organisation. If the overall score $>=7$, then the status of the factor will be noted as "Strong" else will marked as "Weak".

- To assess the feedback, get at the feedback session with participants at case study in order to assure whether OCMM needs any improvement.

The goals of feedback session are:

1) To estimate whether OCMM can be used for the recognition of strong and weak factors in the organisations.

2) To find out whether OCMM is clear, easy to use and especially helpful in evaluating the organisation for outsourcing contract management.

3) To confirm whether the participants satisfied with the assessment results and overall performance of the OCMM.

4) To verify whether the solutions identified for each CSF and CB are easy to use and univocal.

5) To confirm generalisation and applicability of OCMM to software development outsourcing organisations.

6) To check the capacity of OCMM of influencing solid and weak OSDO activities at vendor organisation. 


\section{Reporting}

The target participants for the study are offshore software development outsourcing organisations, practitioners and researchers.

The derived information from the participants will assist outsourcing organisations (vendors) in assessing their strength and weakness in terms of developing, implementing and evaluating proper strategies to afford their software development outsourcing activities. The researcher will publish these outcomes in thesis, journal articles and conference subject to confidentiality issues of maintaining secrecy for individuals and organisations. Furthermore, the data collected during the feedback session discussed in Section 2.3 will assist the researcher to decide whether OCMM requires any advancement.

\section{Timetable}

The timetables for the case studies are given below. There are no real issues if any of the stages takes longer than expected time.

Table 1 Work Planning

\begin{tabular}{ll}
\hline \hline Steps & Duration \\
\hline Planning & One Week \\
Protocol Development & One month \\
Protocol Evaluation/Implementation & One month \\
Data Analysis & Two weeks \\
Reporting & One month \\
\hline \hline
\end{tabular}

\section{UOM Ethics Requirements}

A prior approval of an ethics application was taken from the Ethics Review Panel at University of Malakand, Pakistan. The project was approved by the Advance Study Research Board (ASRB) UOM. The researchers planned the case studies' interviews to meet the ethics requirements, such as "protection of subjects from any damage, deception and loss of privacy". The self-respect and interest of participants will be respected all the times. An approval of the host organisations was derived prior to conduct the research. The information collected through case studies from the organisations will be only used for research purposes. Such information will be treated in the "STRICTEST CONFIDENCE and any publication from this study will present information in aggregate form such that individual organisations or individual respondents participating in the research cannot be identified". The participant is allowed to withdraw their participation any time during this research project. Moreover, only the supervisory team and Mr. Abdul Wahid Khan will have approach to the data. Furthermore, participants will be communicated about the nature of the research, through detailed support prior to the conduct of the study.

\section{Potential Conflict of Interest}

Not known

\section{Divergences}

In case of divergence from the protocol, which may take place during the study; will note down any change in a new Appendix to this document?

\section{Protocol Reviews and Validations}


The protocol was initially reviewed by my supervisor and changes were made as suggested by my supervisor. It was also present to Software Engineering Research Group SERG_UOM for further validation.

\section{References}

[1] Khan, S. U. (2011). Software outsourcing vendors' readiness model. PhD thesis, School of Computing and Maths Keele, UK, 2011.

[2] Niazi, M., Cox, K., \& Verner, J. (2005). An empirical study identifying high perceived value requirements engineering practices. Proceedings of the Fourteen International Conference on Information Systems Development (ISD '2005).

[3] Niazi, M., Wilson, D., \& Zowghi, D. (2005). A framework for assisting the design of effective software process improvement implementation strategies. Journal of Systems and Software, 78, 204-222.

[4] Niazi, M., Wilson, D., \& Zowghi, D. (2005). A maturity model for the implementation of software process improvement: An empirical study. Journal of Systems and Software, 74, 155-172, .

[5] Davis, F. D. (1989) Perceived usefulness, perceived ease of use, and user acceptance of information technology. MIS Quarterly, 13, pp. 319-340.

[6] Davis, F. D., Bagozzi, R. P., \& Warshaw, P. R. (1989). User acceptance of computer technology: A comparison of two theoretical models. Management Science, 35, 982-1003.

[7] Kitchenham, B., \& Charters, C. (2007) Guidelines for performing systematic literature reviews in software engineering. Keele University and Durham University Joint Report.

[8] Barbara, K., \& Pearl, B. (2013). A systematic review of systematic review process research in software engineering. Information and Software Technology, 55, 2049-2075.

[9] Rockart, J. F. (1979). Chief executives define their own data needs. Harvard Business Review, 81-93.

[10] Khan, A. W., \& Khan, S. U. (2013) Critical success factors for offshore software outsourcing contract management from vendors' perspective: An exploratory study using a systematic literature review. IET Software, 7, 327-338.

[11] Khan, A. W., \& Khan, S. U. (2014) Critical challenges in execution of offshore software outsourcing contract from vendors' perspective: A systematic literature review. Proceedings of the 5th International Conference on Information and Communication Systems (ICICS-2014) of IEEE, Jordan University of Science \& Technology (JUST).

[12] Niazi, M. (2004) A framework for assisting the design of effective software process improvement implementation strategies. PhD thesis, University of Technology Sydney.

[13] Yin, R. K. (2009) Case Study Research: Design and Methods. Sage Publications.

[14] Cooper, D., \& Schindler, P. (2001). Business research methods. McGraw-Hill.

1.

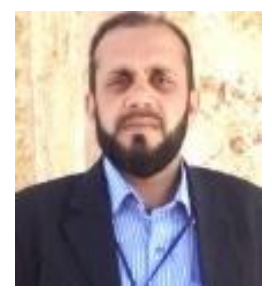

Abdul Wahid Khan working as assistant professor in Department of Computer Science, University of Science \& Technology Bannu, KPK, Pakistan since 2008. He did his PhD in empirical software engineering. He has published number of papers in national and international journal and conferences. The interest area of research is empirical software engineering.

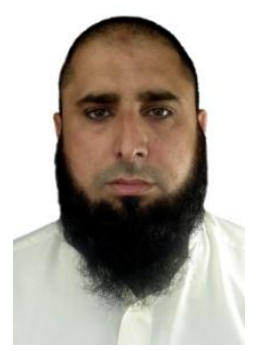

Siffat Ullah Khan is currently working as assistant professor in computer science at University of Malakand, Pakistan. He did his PhD from Keele University UK, 2011. He has a contributed a lot to software engineering community in the areas of global software 
engineering, empirical software engineering, software outsourcing, cloud computing and green IT. He has published more than 100 research articles in peer reviewed international journals and conferences.

Faheem Khan is working as assistant professor in the Department of Computer Science, Bacha Khan University, KPK Pakistan. He did his PhD from University of Malakand at 2016. He is very dedicative researcher and has a lot of contribution in his specialized area. 